\title{
Preventive effects of cilostazol against the development of shunt-dependent hydrocephalus after subarachnoid hemorrhage
}

\author{
Yoshinari Nakatsuka, MD, ${ }^{1}$ Fumihiro Kawakita, MD, ${ }^{1}$ Ryuta Yasuda, MD, PhD, ${ }^{1}$ \\ Yasuyuki Umeda, MD, PhD, ${ }^{2}$ Naoki Toma, MD, PhD, ${ }^{1}$ Hiroshi Sakaida, MD, PhD, ${ }^{3}$ and \\ Hidenori Suzuki, MD, PhD, on behalf of the Prospective Registry for Searching Mediators of \\ Neurovascular Events After Aneurysmal Subarachnoid Hemorrhage (pSEED) Group
}

Departments of ${ }^{1}$ Neurosurgery and ${ }^{3}$ Innovative Neuro-Intervention Radiology, Mie University Graduate School of Medicine; and ${ }^{2}$ Center for Vessels and Heart, Mie University Hospital, Tsu, Japan

\begin{abstract}
OBJECTIVE Chronic hydrocephalus develops in association with the induction of tenascin-C (TNC), a matricellular protein, after aneurysmal subarachnoid hemorrhage (SAH). The aim of this study was to examine if cilostazol, a selective inhibitor of phosphodiesterase Type III, suppresses the development of chronic hydrocephalus by inhibiting TNC induction in aneurysmal SAH patients.
\end{abstract}

METHODS The authors retrospectively reviewed the factors influencing the development of chronic shunt-dependent hydrocephalus in 87 patients with Fisher Grade $3 \mathrm{SAH}$ using multivariate logistic regression analyses. Cilostazol (50 or $100 \mathrm{mg}$ administered 2 or 3 times per day) was administered from the day following aneurysmal obliteration according to the preference of the attending neurosurgeon. As a separate study, the effects of different dosages of cilostazol on the serum TNC levels were chronologically examined from Days 1 to 12 in 38 SAH patients with Fisher Grade 3 SAH.

RESULTS Chronic hydrocephalus occurred in 12 of 36 (33.3\%), 5 of 39 (12.8\%), and 1 of $12(8.3 \%)$ patients in the 0 $\mathrm{mg} / \mathrm{day}, 100$ to $200 \mathrm{mg} / \mathrm{day}$, and $300 \mathrm{mg} / \mathrm{day}$ cilostazol groups, respectively. The multivariate analyses showed that older age (OR 1.10,95\% Cl 1.13-1.24; $p=0.012)$, acute hydrocephalus (OR 23.28, 95\% Cl 1.75-729.83; $p=0.016)$, and cilostazol (OR $0.23,95 \% \mathrm{Cl} 0.05-0.93 ; p=0.038)$ independently affected the development of chronic hydrocephalus. Higher dosages of cilostazol more effectively suppressed the serum TNC levels through Days 1 to 12 post-SAH.

CONCLUSIONS Cilostazol may prevent the development of chronic hydrocephalus and reduce shunt surgery, possibly by the inhibition of TNC induction after SAH.

https://thejns.org/doi/abs/10.3171/2016.5.JNS152907

KEY WORDS cilostazol; hydrocephalus; subarachnoid hemorrhage; tenascin-C; vascular disorders

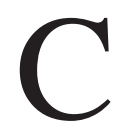
HRONIC shunt-dependent hydrocephalus occurs in $8.9 \%$ to $48 \%$ of patients with aneurysmal subarachnoid hemorrhage (SAH). ${ }^{6}$ Post-SAH chronic hydrocephalus is frequently associated with poor neurological outcomes, which are not completely reversed after CSF shunting. ${ }^{38}$ A number of retrospective studies have identified factors that are predictive of SAH-associated shuntdependent chronic hydrocephalus. ${ }^{6}$ Fenestration of the lamina terminalis during clipping surgery has been suggested to reduce the incidence of shunt-dependent chronic hydrocephalus, but the effects are conflicting. ${ }^{6}$ As far as we know, there are no established treatments that prevent post-SAH chronic hydrocephalus.

The etiology of post-SAH chronic hydrocephalus is still uncertain, but it is most plausible that post-SAH inflammatory reactions or blood-clotting products trigger the proliferation of arachnoid cells and leptomeningeal fibrosis, which impair CSF circulation and/or reabsorption through the arachnoid villi and/or other routes, thereby causing chronic hydrocephalus., $3,20,27$ A previous study reported the possible involvement of tenascin-C (TNC), a matricellular protein, in the development of post-SAH

ABBREVIATIONS cAMP = cyclic adenosine monophosphate; IL = interleukin; MAPK = mitogen-activated protein kinase; PDGF = platelet-derived growth factor; pSEED = Prospective Registry for Searching Mediators of Neurovascular Events After Aneurysmal Subarachnoid Hemorrhage; SAH = subarachnoid hemorrhage; TGF = transforming growth factor; TNC = tenascin-C; TNF = tumor necrosis factor; WFNS = World Federation of Neurosurgical Societies.

SUBMITTED December 13, 2015. ACCEPTED May 18, 2016.

INCLUDE WHEN CITING Published online August 5, 2016; DOI: 10.3171/2016.5.JNS152907. 
chronic hydrocephalus. ${ }^{32} \mathrm{TNC}$ is induced by inflammation and promotes cell proliferation and fibrosis. ${ }^{5,15}$ TNC was reported to be induced in the serum and CSF after aneurysmal SAH. ${ }^{30,31}$ In a clinical setting, higher TNC levels in the CSF were observed in SAH patients with worse neurological grades on admission, more massive hemorrhage on CT, symptomatic vasospasm, and worse outcomes, in addition to chronic hydrocephalus, while patient age did not affect TNC levels. ${ }^{30,32}$ TNC also caused brain injuries after experimental SAH. ${ }^{12,29}$

Cilostazol-a selective inhibitor of phosphodiesterase Type III and a cyclic adenosine monophosphate (cAMP)elevating agent-is a clinically available antiplatelet agent and has pleiotropic actions, including vasodilatory and antiinflammatory effects. ${ }^{25}$ It has been suggested that cilostazol reduces symptomatic vasospasm and is relatively widely administered to SAH patients in Japan. ${ }^{28}$ Cilostazol has also been reported to inhibit TNC induction in cultured vascular smooth muscle cells. ${ }^{13}$ Therefore, we speculated that cilostazol might prevent the occurrence of chronic hydrocephalus by inhibiting TNC induction after $\mathrm{SAH}$. Thus, the goal of this study was to evaluate if the administration of cilostazol is a suppressing factor against the occurrence of chronic shunt-dependent hydrocephalus and if oral cilostazol inhibits serum TNC induction after aneurysmal SAH.

\section{Methods}

\section{Retrospective Study to Determine Factors Related to Shunt-Dependent Hydrocephalus After SAH}

This study was approved by the ethics committee at our institution. We reviewed the medical records of $153 \mathrm{SAH}$ patients who were admitted to our hospital between January 2007 and January 2015. All neuroimages were reassessed using the Fisher scale, ${ }^{11}$ and rebleeding, the development of cerebral infarction, and other clinical variables were assessed by an experienced neurosurgeon (F.K.) who was unaware of other clinical data. As a result, 87 patients met the following inclusion criteria: $\geq 20$ years of age at onset, SAH classified as Fisher Grade 3 on admission CT scans, ${ }^{11}$ saccular aneurysm as the cause of SAH confirmed on digital subtraction angiography, aneurysmal obliteration by clipping or coiling performed within 14 days of onset, and survival of more than 14 days after onset. Patients with dissecting, traumatic, mycotic, and arteriovenous malformation-related aneurysms or SAH of an unknown etiology were excluded from the study. After angiographic confirmation of the aneurysm, surgical clipping or endovascular coiling of the lesion was performed as deemed appropriate for the individual patient by the attending neurosurgeon. A ventricular catheter was placed in all patients with ventriculomegaly and a decreased level of consciousness that could not be attributed to causes other than acute hydrocephalus at admission. In other cases, according to the preference of the attending neurosurgeons, a ventricular drain was placed to control brain swelling, and a cisternal drain was placed in the basal cistern to promote $\mathrm{SAH}$ clearance after surgical clipping. If progressive ventriculomegaly was observed postoperatively and within 14 days of onset, a lumbar spinal drain was placed irrespec- tive of clipping or coiling. Drainage was continued for 7 to 14 days, and the volume of drained CSF was maintained at 150 to $250 \mathrm{ml} /$ day by changing the height of the drainage siphon. All patients without renal dysfunction received intravenous fasudil hydrochloride $(30 \mathrm{mg}$ administered twice per day; Asahi Kasei Pharma Co.) from Day 1 postclipping or postcoiling to Day 14 posthemorrhage. In addition, oral or enteral cilostazol $(50$ or $100 \mathrm{mg}$ administered 2 or 3 times per day; i.e., 100, 150, 200, or $300 \mathrm{mg} / \mathrm{day}$; Otsuka Pharmaceutical Co.) was administered from Day 1 postclipping or postcoiling to Day 14 posthemorrhage or later according to the preference of the attending neurosurgeons. For at least 14 days after SAH, patients were managed in the intensive care unit, vital signs and laboratory data were assessed, and antibiotics were administered while the CSF drain was in place in order to maintain normovolemia; prevent meningitis, pneumonia, and hypoxia; and correct anemia and hypoproteinemia. All patients with symptomatic vasospasm-as defined as otherwise unexplained clinical deterioration (i.e., a new focal deficit, a decrease in the level of consciousness, or both), a new cerebral infarction (delayed cerebral infarction) on CT that was not visible on the admission or immediate postoperative scan, or both-were treated with hypertensive hypervolemic therapy and/or endovascular therapy (intraarterial fasudil hydrochloride infusions or angioplasty). Other potential causes of clinical deterioration, such as hydrocephalus, rebleeding, or seizures, were rigorously excluded. Chronic hydrocephalus was diagnosed when clinical deterioration with no detectable causes other than hydrocephalus occurred after Day 14 posthemorrhage and when the ventricular size progressively increased and the Evans Index became greater than $0.30 .{ }^{35}$ Chronic hydrocephalus was treated with ventriculoperitoneal shunting. Clinical outcome was evaluated using the modified Rankin Scale at discharge. Clinical variables-including age, sex, World Federation of Neurosurgical Societies (WFNS) grade at admission and before any treatment, ${ }^{8}$ the modality used for aneurysmal obliteration, location of the ruptured aneurysm, acute hydrocephalus requiring ventricular drainage at admission, placement of lumbar drainage catheters the day after aneurysmal obliteration (or later), placement of other CSF drains, cilostazol administration, and the presence of symptomatic vasospasm, delayed cerebral infarction, and shunt-dependent hydrocephalus-were recorded, and factors influencing the development of shunt-dependent hydrocephalus were determined using univariate and multivariate analyses.

\section{Prospective Study to Determine if Cilostazol Suppresses Serum TNC Levels After SAH}

After searching for mediators of neurovascular events following aneurysmal $\mathrm{SAH}$, we identified 90 patients registered in the Prospective Registry for Searching Mediators of Neurovascular Events After Aneurysmal Subarachnoid Hemorrhage (pSEED) who were treated at 9 tertiary referral centers in the Mie prefecture in Japan (Appendix) between September 2013 and October 2014. The inclusion criteria were as follows: $\geq 20$ years of age at onset, SAH identified on CT scans or lumbar puncture, saccular aneurysm as the cause of SAH and confirmed on 
digital subtraction angiography, and aneurysmal obliteration by clipping or coiling within 48 hours of onset. The timing of aneurysmal obliteration, selection of clipping or coiling, and other medical management or treatment strategies were decided by the onsite investigators and not limited. After written informed consent was obtained, clinical variables were recorded as in the retrospective study. After CT confirmation of no complications occurring the day after clipping or coiling, blood samples were serially collected with minimal stasis from a vein on Days 1 to 3 , Days 4 to 6 , Days 7 to 9 , and Days 10 to 12 after onset. All samples were centrifuged for 5 minutes at $3000 g$, and the supernatant fluid was stored at $-30^{\circ} \mathrm{C}$ until assayed. Among these patients, 38 were selected for the study because they had SAH of Fisher Grade 3 status at admission and survived for more than 14 days post-SAH with neither angiographic complications and surgical complications nor concomitant inflammatory, malignant, and other diseases that are known to upregulate TNC. ${ }^{5,30-32}$ The serum TNC concentrations were determined using a commercially available enzyme-linked immunosorbent assay kit for high-molecular-weight human TNC variants (code no. 27767; IBL).

\section{Statistical Analysis}

There were no missing data in the patients who met the inclusion criteria in both the retrospective and prospective studies. Variables were recorded as categorical or continuous variables. Categorical variables were reported as the number and percentage and analyzed using the chi-square or Fisher exact test, as appropriate. Continuous variables were reported as the mean \pm standard deviation and compared between 2 groups using the unpaired t-test. If significant variance was found, intergroup comparisons among 3 groups were determined by 1-way ANOVA and then the Tukey-Kramer multiple comparison procedure $(95 \%$ CI). The impact of each variable on the development of shunt-dependent hydrocephalus was determined by multivariate unconditional logistic regression analysis using shunt-dependent hydrocephalus (presence or absence) as the dependent variable. All variables were considered independent variables irrespective of significance in the univariate analysis, although only the variable with the smallest probability value was used as a candidate variable among similar clinical variables that were intercorrelated. The adjusted ORs with 95\% CIs were calculated, and the independence of the variables was tested using the likelihood ratio test on reduced models. A $p$ value $\leq 0.05$ was considered significant.

\section{Results}

\section{Factors Related to Shunt-Dependent Hydrocephalus After SAH}

Of the 87 patients (mean age $63.6 \pm 12.9$ years), 63 patients were female and 24 patients were male. Eighteen patients underwent ventriculoperitoneal shunting at $36.7 \pm$ 18.2 days after SAH. There were no significant differences between the shunt-treated and non-shunt-treated groups in terms of patient sex, WFNS grade on admission, aneurysm location, and treatment modality. The univariate analyses showed that older age, acute hydrocephalus, CSF drainage, symptomatic vasospasm, and no cilostazol use were significant factors for the development of shunt-dependent hydrocephalus (Table 1). Head CT findings were assessed before clipping or coiling, at 12 to 24 hours after clipping or coiling, and on Days 3 to 4, Days 7 to 10, and Days 14 to 21 in all patients, which revealed that delayed cerebral infarction occurred in 6 of 18 shunt-treated patients (33.3\%) and 12 of 69 non-shunt-treated patients (17.4\%), but the difference did not reach statistical significance (Table 1). A cisternal drain was placed at the time of clipping in 3 patients, 2 of whom were treated with 0 or $200 \mathrm{mg} /$ day cilostazol and did not undergo eventual shunting, while the other patient was treated with $100 \mathrm{mg} /$ day cilostazol and underwent shunting. Shunt-dependent hydrocephalus was associated with worse outcomes.

Multivariate analyses were performed using all of the variables listed in Table 1 (except the use of CSF drains, most of which were placed to treat delayed progressive ventriculomegaly and therefore highly correlated with the development of shunt-dependent hydrocephalus and the modified Rankin Scale at discharge), although only the variable with the smallest probability value was used as a candidate variable among similar and intercorrelated clinical variables. The multivariate analyses revealed that older age (OR 1.10, 95\% CI 1.13-1.24; $\mathrm{p}=0.012)$ and acute hydrocephalus (OR 23.28, 95\% CI 1.75-729.83; $\mathrm{p}=0.016$ ) were independent predictive factors for the development of shunt-dependent hydrocephalus, while cilostazol (OR $0.23,95 \%$ CI $0.05-0.93 ; \mathrm{p}=0.038$ ) independently suppressed it.

Regarding the cilostazol dosage, 12 of 36 patients $(33.3 \%)$ treated with no cilostazol eventually needed shunting, while the 100 to $200 \mathrm{mg} /$ day and $300 \mathrm{mg} / \mathrm{day}$ cilostazol groups required less shunting (5 of 39 patients [12.8\%] in the 100-200 mg/day cilostazol group, and 1 of 12 patients [8.3\%] in the $300 \mathrm{mg}$ /day cilostazol group). The incidence of shunt-dependent hydrocephalus was significantly lower in patients treated with cilostazol than patients treated without cilostazol (Fig. 1). There were no significant differences in the clinical features among the patients treated with different cilostazol doses, except for the incidence of shunt-dependent hydrocephalus (Table 2). Adverse events due to cilostazol occurred in 2 patients in the $200 \mathrm{mg} /$ day cilostazol group: in 1 patient, the dose of cilostazol was reduced to $100 \mathrm{mg}$ /day due to headache, and in the other patient cilostazol was discontinued on Day 2 post-SAH due to severe headache. Tachycardia or hemorrhagic complications affecting cilostazol usage did not occur.

\section{Effects of Cilostazol on Serum TNC Levels After SAH}

Of 38 patients studied prospectively, 5 patients were treated without cilostazol, 23 patients were treated with 100 to $200 \mathrm{mg} /$ day cilostazol, and 10 patients were treated with $300 \mathrm{mg} /$ day cilostazol. There were no adverse events such as headache, tachycardia, or hemorrhagic complications that affected cilostazol usage. The $300 \mathrm{mg} /$ day cilostazol group underwent endovascular coiling of aneurysms most frequently, but the other clinical features were not significantly different among the 3 groups (Table 3 ). The inci- 
TABLE 1. Clinical features in patients with and without shunt-dependent hydrocephalus after SAH*

\begin{tabular}{|c|c|c|c|c|}
\hline Variable & Total & w/o Hydrocephalus & w/ Hydrocephalus & p Value† \\
\hline No. of patients & 87 & 69 & 18 & \\
\hline Mean age $\pm S D$, yrs & $63.6 \pm 12.9$ & $61.7 \pm 12.9$ & $70.6 \pm 10.7$ & 0.010 \\
\hline Sex & & & & 0.568 \\
\hline Female & $63(72.4)$ & $49(71.0)$ & $14(77.8)$ & \\
\hline Male & $24(27.6)$ & $20(29.0)$ & $4(22.2)$ & \\
\hline Admission WFNS grade & & & & 0.137 \\
\hline I-III & $52(59.8)$ & $44(63.8)$ & $8(44.4)$ & \\
\hline IV-V & $35(40.2)$ & $25(36.2)$ & $10(55.6)$ & \\
\hline Aneurysm location & & & & 0.980 \\
\hline $\mathrm{ACoA}$ & $27(31.0)$ & $21(30.4)$ & $6(33.3)$ & \\
\hline ICA & $32(36.8)$ & $26(37.7)$ & $6(33.3)$ & \\
\hline MCA & $15(17.2)$ & $11(15.9)$ & $4(22.2)$ & \\
\hline Posterior circulation & $5(5.7)$ & $5(7.2)$ & $0(0)$ & \\
\hline Other & $8(9.2)$ & $6(8.7)$ & $2(11.1)$ & \\
\hline Treatment modality & & & & 0.120 \\
\hline Endovascular coiling & $57(65.5)$ & $48(69.6)$ & $9(50.0)$ & \\
\hline Surgical clipping & $30(34.5)$ & $21(30.4)$ & $9(50.0)$ & \\
\hline Acute hydrocephalus & $6(6.9)$ & $2(2.9)$ & $4(22.2)$ & 0.004 \\
\hline CSF drainage & $20(23.0)$ & $9(13.0)$ & $11(61.1)$ & $<0.0001$ \\
\hline Lumbar drainage & $12(13.8)$ & $4(5.8)$ & $8(44.4)$ & $<0.0001$ \\
\hline Other drainage & $9(10.3)$ & $5(7.2)$ & $4(22.2)$ & 0.063 \\
\hline Symptomatic vasospasm & $23(26.4)$ & $14(20.3)$ & $9(50.0)$ & 0.011 \\
\hline Delayed cerebral infarct & $18(20.7)$ & $12(17.4)$ & $6(33.3)$ & 0.137 \\
\hline Cilostazol & $51(58.6)$ & $45(65.2)$ & $6(33.3)$ & 0.014 \\
\hline mRS Score $0-2$ at discharge & $49(56.3)$ & $47(68.1)$ & $2(11.1)$ & $<0.0001$ \\
\hline
\end{tabular}

dence of shunt-dependent hydrocephalus was lower in the $300 \mathrm{mg} /$ day cilostazol group (1 of 10 patients; $10.0 \%$ ) compared with the $0 \mathrm{mg} /$ day ( 2 of 5 patients; $40.0 \%$ ) and 100 to $200 \mathrm{mg}$ /day cilostazol groups (8 of 23 patients; $34.8 \%$ ), but the difference did not reach statistical significance due to the limited number of patients. The administration of 300 $\mathrm{mg} /$ day cilostazol significantly decreased the serum TNC levels compared with the $0 \mathrm{mg} / \mathrm{day}$ and 100 to $200 \mathrm{mg} / \mathrm{day}$ cilostazol groups through Days 1 to 12 (Fig. 2), irrespective of WFNS grade on admission (Table 4).

\section{Discussion}

This is the first study to demonstrate that the administration of cilostazol is an independent factor that reduces the development of chronic shunt-dependent hydrocephalus after SAH. In addition, a higher dosage of cilostazol suppressed the serum TNC levels more. These results suggest that cilostazol would be the first treatment to prevent chronic shunt-dependent hydrocephalus, possibly by suppressing TNC induction.

The key mechanisms that disrupt CSF homeostasis and the leptomeninges (the arachnoid mater and pia mater) forming the subarachnoid space may be mediated by an inflammatory reaction and blood-clotting products. A rap-

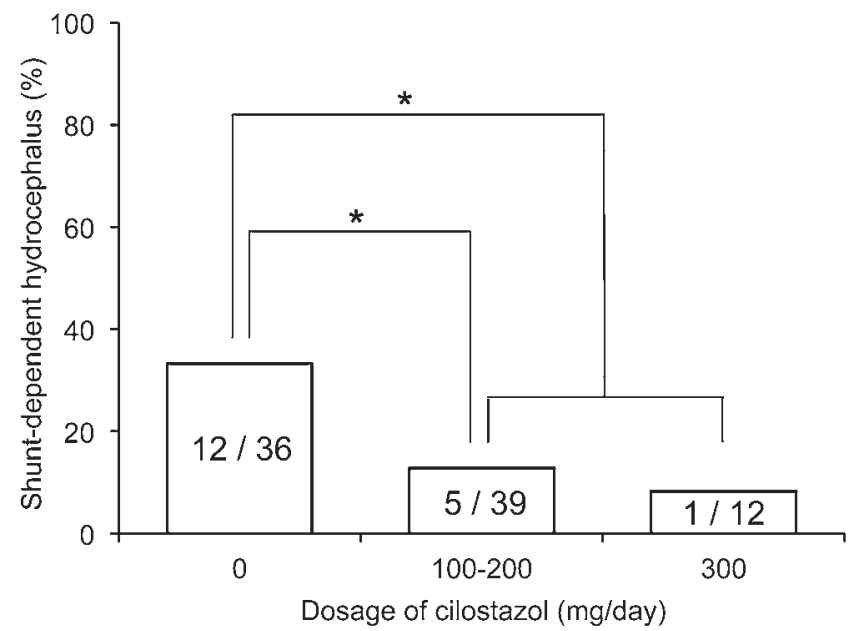

FIG. 1. The incidence of shunt-dependent hydrocephalus among patients treated with $0 \mathrm{mg} / \mathrm{day}, 100$ to $200 \mathrm{mg} /$ day, and $300 \mathrm{mg} /$ day cilostazol. The numbers depicted in the bar express the number of shunt-treated patients divided by the total number of patients in the group. Cilostazol usage significantly decreases the incidence of shuntdependent hydrocephalus. ${ }^{*} p<0.05$, Fisher exact test. 
TABLE 2. Clinical features in SAH patients treated with different cilostazol doses in the retrospective study*

\begin{tabular}{|c|c|c|c|}
\hline \multirow[b]{2}{*}{ Variable } & \multicolumn{3}{|c|}{ Cilostazol Dose (mg/day) } \\
\hline & 0 & $100-200$ & 300 \\
\hline No. of patients & 36 & 39 & 12 \\
\hline Mean age $\pm S D$, yrs & $65.6 \pm 13.3$ & $61.1 \pm 12.9$ & $65.4 \pm 10.4$ \\
\hline \multicolumn{4}{|l|}{ Sex } \\
\hline Female & $24(66.7)$ & $30(76.9)$ & $9(75.0)$ \\
\hline Male & $12(33.3)$ & $9(23.1)$ & $3(25.0)$ \\
\hline \multicolumn{4}{|l|}{ Admission WFNS grade } \\
\hline$|-|||$ & $20(55.6)$ & $24(61.5)$ & $8(66.7)$ \\
\hline IV-V & $16(44.4)$ & $15(38.5)$ & $4(33.3)$ \\
\hline \multicolumn{4}{|l|}{ Aneurysm location } \\
\hline $\mathrm{ACoA}$ & $10(27.8)$ & $11(28.2)$ & $6(50.0)$ \\
\hline ICA & $16(44.4)$ & $13(33.3)$ & $3(25.0)$ \\
\hline MCA & $5(13.9)$ & $9(23.1)$ & $1(8.3)$ \\
\hline Posterior circulation & $1(2.8)$ & $3(7.7)$ & $1(8.3)$ \\
\hline Other & $4(11.1)$ & $3(7.7)$ & $1(8.3)$ \\
\hline \multicolumn{4}{|l|}{ Treatment modality } \\
\hline Endovascular coiling & $22(61.1)$ & $26(66.7)$ & $9(75.0)$ \\
\hline Surgical clipping & $14(38.9)$ & $13(33.3)$ & $3(25.0)$ \\
\hline Acute hydrocephalus & $3(8.3)$ & $2(5.1)$ & $1(8.3)$ \\
\hline CSF drainage & $12(33.3)$ & $6(15.4)$ & $2(16.7)$ \\
\hline Lumbar drainage & $7(19.4)$ & $3(7.7)$ & $2(16.7)$ \\
\hline Other drainage & $6(16.7)$ & $3(7.7)$ & $0(0)$ \\
\hline Symptomatic vasospasm & $12(33.3)$ & $9(23.1)$ & $2(16.7)$ \\
\hline Delayed cerebral infarction & $10(27.8)$ & $7(17.9)$ & $1(8.3)$ \\
\hline $\begin{array}{l}\text { Shunt-dependent hydro- } \\
\text { cephalus }\end{array}$ & $12(33.3)$ & $5(12.8) \dagger$ & $1(8.3)$ \\
\hline mRS score $0-2$ at discharge & $17(47.2)$ & $24(61.5)$ & $8(66.7)$ \\
\hline
\end{tabular}

* Data are presented as the number of patients (\%) unless stated otherwise. $\dagger p<0.05$ versus the $0 \mathrm{mg} /$ day cilostazol group according to the Fisher exact test.

id inflammatory cell response occurs in the leptomeninges after $\mathrm{SAH}$, with polymorphonuclear cells dominating during the first 24 hours and mononuclear cells thereafter. ${ }^{26,33}$ These inflammatory cells secrete cytokines that trigger a fibroproliferative reaction by acting as mitogens and chemoattractants for fibroblasts. ${ }^{17,18}$ Reportedly, inflammatory cytokines and growth factors such as tumor necrosis factor (TNF)- $\alpha$, interleukin (IL)-1, IL-6, platelet-derived growth factor (PDGF), and transforming growth factor (TGF) $-\beta$ are upregulated in the acute stage of SAH., ${ }^{2,919,21,40}$ Thrombin is also released by the blood-clotting cascade in the CSF of patients with SAH..$^{34}$ Thrombin, TGF $\beta$, and PDGF promoted human leptomeningeal cell proliferation in vitro, ${ }^{23}$ and hydrocephalus developed in mice after an intracerebral injection of TGF $\beta,{ }^{36}$ a potent fibrogenic cytokine. CSF studies in humans have suggested that leptomeningeal collagen synthesis was induced within the first 48 to 72 hours after SAH. ${ }^{27}$ Autopsy studies have shown blood and inflammatory cell accumulation within the arachnoid villi during the 1st week, greater mitotic activity and the proliferation of leptomeningeal cells during the 2 nd and
TABLE 3. Clinical features in SAH patients treated with different cilostazol doses in the prospective registry study*

\begin{tabular}{|c|c|c|c|}
\hline \multirow[b]{2}{*}{ Variable } & \multicolumn{3}{|c|}{ Cilostazol Dose (mg/day) } \\
\hline & 0 & $100-200$ & 300 \\
\hline No. of patients & 5 & 23 & 10 \\
\hline Mean age $\pm S D$, yrs & $51.6 \pm 8.1$ & $65.5 \pm 14.4$ & $64.1 \pm 12.3$ \\
\hline \multicolumn{4}{|l|}{ Sex } \\
\hline Female & $3(60.0)$ & $10(43.5)$ & $7(70.0)$ \\
\hline Male & $2(40.0)$ & $13(56.5)$ & $3(30.0)$ \\
\hline \multicolumn{4}{|l|}{ Admission WFNS grade } \\
\hline$|-|||$ & $4(80.0)$ & $11(47.8)$ & $7(70.0)$ \\
\hline IV-V & $1(20.0)$ & $12(52.2)$ & $3(30.0)$ \\
\hline \multicolumn{4}{|l|}{ Aneurysm location } \\
\hline ACoA & $2(40.0)$ & $5(21.7)$ & $4(40.0)$ \\
\hline ICA & $1(20.0)$ & $11(47.8)$ & $3(30.0)$ \\
\hline MCA & $1(20.0)$ & $3(13.0)$ & $1(10.0)$ \\
\hline Posterior circulation & $1(20.0)$ & $3(13.0)$ & $2(20.0)$ \\
\hline Other & $0(0)$ & $1(4.3)$ & $0(0)$ \\
\hline \multicolumn{4}{|l|}{ Treatment modality } \\
\hline Endovascular coiling & $1(20.0)$ & $1(4.3)$ & $8(80.0) \dagger \ddagger$ \\
\hline Surgical clipping & $4(80.0)$ & $22(95.7)$ & $2(20.0)$ \\
\hline Acute hydrocephalus & $1(20.0)$ & $13(56.5)$ & $3(30.0)$ \\
\hline CSF drainage & $1(20.0)$ & $8(34.8)$ & $2(20.0)$ \\
\hline Lumbar drainage & $0(0)$ & $5(21.7)$ & $2(20.0)$ \\
\hline Other drainage & $1(20.0)$ & $4(17.4)$ & $0(0)$ \\
\hline Symptomatic vasospasm & $2(40.0)$ & $4(17.4)$ & $0(0)$ \\
\hline Delayed cerebral infarction & $1(20.0)$ & $3(13.0)$ & $0(0)$ \\
\hline $\begin{array}{l}\text { Shunt-dependent hydro- } \\
\text { cephalus }\end{array}$ & $2(40.0)$ & $8(34.8)$ & $1(10.0)$ \\
\hline $\begin{array}{l}\text { mRS score } 0-2 \text { at dis- } \\
\text { charge }\end{array}$ & $3(60.0)$ & $6(26.1)$ & $6(60.0)$ \\
\hline
\end{tabular}

* Data are presented as the number of patients (\%) unless stated otherwise. $\dagger p<0.0001$ versus the 100 to $200 \mathrm{mg} /$ day cilostazol group.

$\ddagger p<0.05$ versus the $0 \mathrm{mg} /$ day cilostazol group according to the Fisher exact test.

3rd weeks, and dense collagen fibers in the subarachnoid space in the 4th week after SAH..$^{20,22}$ Impaired CSF circulation and the occlusion of CSF drainage channels by cell proliferation and collagen deposits may cause chronic shunt-dependent hydrocephalus, but the initial reactions are induced acutely after SAH. In this study, cilostazol was administered from the day following aneurysmal obliteration, usually within 48 to 72 hours after onset, to Day 14 post-SAH or later. The administration timing and period of cilostazol use may be reasonable to prevent chronic hydrocephalus, but further studies are needed to determine the optimal protocol, including the dosage of cilostazol and the timing and period of administration.

The expression of TNC is extremely limited in normal adult tissues, but is spatiotemporally upregulated by various pro- and antiinflammatory cytokines, ${ }^{5,13}$ including TNF $\alpha$, IL-1, PDGF, and TGF $\beta$, which are upregulated after SAH. ${ }^{2,9,19,40}$ Since TNC is reported to regulate the cell phenotype and promote the migration and proliferation of 


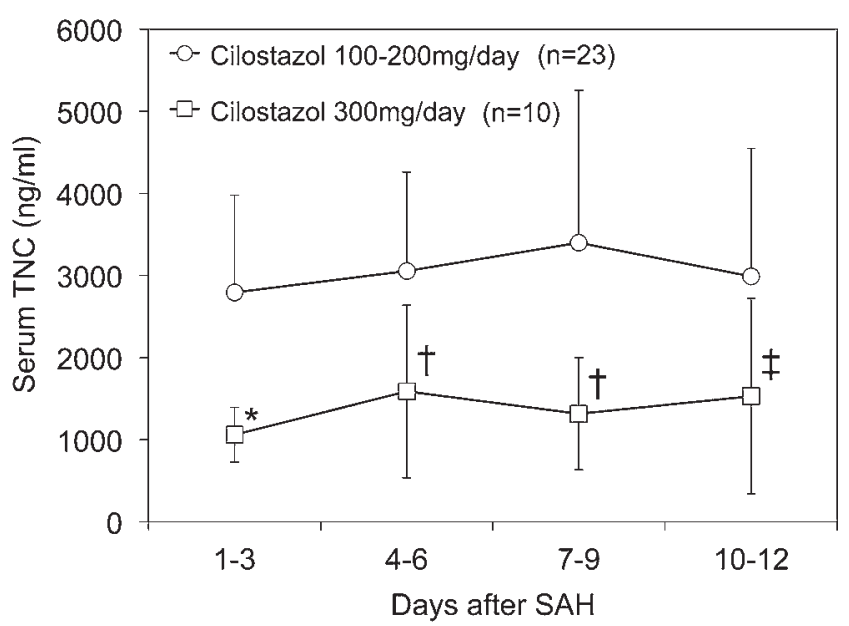

FIG. 2. Serum TNC concentrations after cilostazol treatment. Data represent the mean \pm standard deviation. Serum TNC concentrations are significantly lower in the $300 \mathrm{mg} /$ day cilostazol group than in the 100 to $200 \mathrm{mg} /$ day cilostazol group. ${ }^{\star} p<0.0005,{ }^{\dagger} p<0.005$, and ${ }^{\ddagger} p<0.05$ according to the unpaired t-test.

myofibroblasts, TNC induction may cause the proliferation of the leptomeningeal cells observed after SAH. ${ }^{5,37}$ TNC also promotes tissue fibrosis by increasing Type I and III collagen synthesis, ${ }^{10}$ and the synergistic effects of TNC and TGF $\beta$-one of the most potent profibrogenic substances - have been reported. ${ }^{16}$ Leptomeningeal cells may therefore become more activated and induce more massive fibrosis in an environment that is rich in TNC. Our previous study demonstrated that SAH patients who eventually needed ventriculoperitoneal shunting had significantly higher TNC levels in their CSF than patients who did not require shunting. ${ }^{32}$ Higher TNC levels in the CSF were also associated with a worse WFNS grade on admission, symptomatic vasospasm, and worse outcomes, but the treatment modality (clipping or coiling) did not affect the TNC levels. ${ }^{30}$ The highest increase in the CSF concentration of TNC was in the first 3 days, which is consistent with a CSF study in humans that suggested that leptomeningeal collagen synthesis was induced within the first 48 to 72 hours after SAH. ${ }^{27}$ TNC might be an important substance that mediates post-SAH inflammation and leptomeningeal fibrosis, leading to the development of post-SAH chronic hydrocephalus. This study suggested that cilo- stazol prevented chronic shunt-dependent hydrocephalus, and the effect was greater with a higher dosage of cilostazol and associated with suppressed serum TNC levels through Days 1 to 12 post-SAH. In a prospective registry study, although cerebral aneurysms in the $300 \mathrm{mg} /$ day cilostazol group were most frequently treated with endovascular coiling, endovascular treatment might not affect TNC levels as our previous study suggested. ${ }^{30}$ Cilostazol is reported to inhibit the proliferation of cultured vascular smooth muscle cells in a concentration-dependent manner: an increase in cilostazol concentration from 100 to 200 $\mu \mathrm{M}$ suppressed the proliferation to one-tenth. ${ }^{39} \mathrm{~A}$ multicenter, randomized, double-blind, placebo-controlled trial also showed that 100 to $200 \mathrm{mg} /$ day cilostazol produces a dose-dependent improvement in the treadmill walking distance in patients with intermittent claudication. ${ }^{1}$ In addition, a Phase I study showed that a single administration of 25 to $300 \mathrm{mg}$ cilostazol dependently increased the blood concentration dose, which peaked after 3 to 4 hours and declined with half-lives of 2.6 to 3.2 hours. ${ }^{24}$ These findings support our result that $300 \mathrm{mg} /$ day cilostazol (100 mg cilostazol administered 3 times per day) suppressed the TNC levels more effectively compared with 100 to 200 $\mathrm{mg}$ /day cilostazol.

TNC has been reported to cause brain injuries such as neuronal apoptosis and blood-brain barrier disruption after experimental SAH. ${ }^{12,29}$ Such brain injuries may decrease brain parenchymal volume and contribute to ventriculomegaly with time, thereby explaining why the poor neurological status and cognitive deficits associated with chronic hydrocephalus may not be completely reversed after CSF shunting. This study also showed that most patients with chronic hydrocephalus had a poor neurological status even after CSF shunting. It will be meaningful to examine if cilostazol can inhibit brain TNC induction and therefore directly suppress brain injury after SAH.

Some Japanese neurosurgeons administer cilostazola selective inhibitor of phosphodiesterase Type III and a cAMP-elevating agent-to prevent cerebral vasospasm in SAH patients. ${ }^{28}$ However, in this study, cilostazol reduced the incidence of chronic shunt-dependent hydrocephalus independent of vasospasm occurrence, possibly by the inhibitory effects on TNC induction. Reportedly, cilostazol completely inhibited TNC induction by PDGF-BB at the transcriptional level in cultured vascular smooth muscle cells. ${ }^{13}$ It has been shown that the cAMP-protein kinase

TABLE 4. Serum TNC levels in SAH patients treated with different cilostazol doses in the prospective registry study*

\begin{tabular}{|c|c|c|c|}
\hline \multirow[b]{2}{*}{ Variable } & \multicolumn{3}{|c|}{ Cilostazol Dose (mg/day) } \\
\hline & 0 & $100-200$ & 300 \\
\hline All cases & $2618 \pm 1230$ & $3154 \pm 1494$ & $1373 \pm 904 \dagger \ddagger$ \\
\hline No. of patients & 5 & 23 & 10 \\
\hline Admission WFNS Grade I-III & $2318 \pm 997$ & $2850 \pm 1156$ & $1572 \pm 1000 \dagger \ddagger$ \\
\hline No. of patients & 4 & 11 & 7 \\
\hline Admission WFNS Grade IV-V & $3820 \pm 1336$ & $3433 \pm 1701$ & $908 \pm 292 \dagger \ddagger$ \\
\hline No. of patients & 1 & 12 & 3 \\
\hline
\end{tabular}


A signaling pathway inhibits the transmission of Ras signals from the plasma membrane by preventing the Rasdependent activation of Raf-1, resulting in the inhibition of mitogen-activated protein kinase (MAPK) activation. ${ }^{7,14}$ MAPK is involved in the induction of TNC, ${ }^{4}$ and thus cilostazol may have inhibitory effects on TNC expression through the cAMP-protein kinase A signaling pathway and its inhibitory effect on the downstream MAPK pathway. However, since cilostazol has pleiotropic actions such as antiinflammatory effects, ${ }^{25}$ we cannot exclude the possibility that cilostazol prevents chronic shunt-dependent hydrocephalus via TNC-unrelated mechanisms.

This study has some limitations. First, the use of cilostazol and its dosages were administered according to physician preference, and therefore there is a concern as to selection bias, although the multivariate analyses showed that cilostazol use was an independent suppressing factor for shunt-dependent hydrocephalus in this study. Second, the numbers of patients who received each different cilostazol dose were small. Third, the diagnosis of chronic hydrocephalus and the decision to shunt were somewhat subjective, and blind evaluation is needed to avoid these issues. To confirm the findings of this study, a large-scale, double-blind, randomized control trial is needed. Lastly, this is a descriptive study. Basic research is also needed to determine the pathophysiological relationships between TNC and chronic hydrocephalus.

\section{Conclusions}

The present study is the first to show the finding that cilostazol can reduce chronic shunt-dependent hydrocephalus after aneurysmal SAH, possibly by suppressing TNC induction. Cilostazol could be a novel therapeutic approach to prevent chronic hydrocephalus after SAH.

\section{Acknowledgments}

This work was supported by a Grant-in-Aid for Scientific Research from the Mie University Hospital Seed Grant Program 2014, SENSHIN Medical Research Foundation (2015-1-03), and the Okasan-Kato Foundation (15-1-58), which were awarded to Dr. Suzuki. We thank Ms. Chiduru Yamamoto, Department of Neurosurgery, Mie University Graduate School of Medicine, for providing technical assistance.

\section{Appendix PSEED Group}

Department of Neurosurgery, Kuwana West Medical Center, Kuwana (Masatoshi Muramatsu, Kazuhide Hamada), Mie Prefectural General Medical Center, Yokkaichi (Yusuke Kamei, Akane Yamamichi, Keiji Fukazawa), Suzuka Kaisei Hospital, Suzuka (Tomohiro Araki, Yoichi Miura), Suzuka General Hospital, Suzuka (Kenji Kawaguchi, Katsuhiro Tanaka), Mie University Hospital, Tsu (Hidenori Suzuki, Takanori Sano, Fumihiro Kawakita, Naoki Ichikawa, Satoru Tanioka), Mie Chuo Medical Center, Tsu (Fujimaro Ishida, Masato Shiba), Matsusaka Chuo General Hospital, Matsusaka (Shigehiko Niwa, Kazuhiko Tsuda), Saiseikai Matsusaka General Hospital, Matsusaka (Hiroto Murata, Masashi Fujimoto), and Ise Red Cross Hospital, Ise (Fumitaka Miya, Hirofumi Nishikawa, Yotaro Kitano).

\section{References}

1. Beebe HG, Dawson DL, Cutler BS, Herd JA, Strandness DE
Jr, Bortey EB, et al: A new pharmacological treatment for intermittent claudication: results of a randomized, multicenter trial. Arch Intern Med 159:2041-2050, 1999

2. Borel CO, McKee A, Parra A, Haglund MM, Solan A, Prabhakar V, et al: Possible role for vascular cell proliferation in cerebral vasospasm after subarachnoid hemorrhage. Stroke 34:427-433, 2003

3. Brinker T, Stopa E, Morrison J, Klinge P: A new look at cerebrospinal fluid circulation. Fluids Barriers CNS 11:10, 2014

4. Chiquet M, Sarasa-Renedo A, Tunç-Civelek V: Induction of tenascin- $\mathrm{C}$ by cyclic tensile strain versus growth factors: distinct contributions by Rho/ROCK and MAPK signaling pathways. Biochim Biophys Acta 1693:193-204, 2004

5. Chiquet-Ehrismann R, Chiquet M: Tenascins: regulation and putative functions during pathological stress. J Pathol 200:488-499, 2003

6. Connolly ES Jr, Rabinstein AA, Carhuapoma JR, Derdeyn CP, Dion J, Higashida RT, et al: Guidelines for the management of aneurysmal subarachnoid hemorrhage: a guideline for healthcare professionals from the American Heart Association/American Stroke Association. Stroke 43:1711-1737, 2012

7. Cook SJ, McCormick F: Inhibition by cAMP of Ras-dependent activation of Raf. Science 262:1069-1072, 1993

8. Drake CG, Hunt WE, Kassell N, Pertuiset B, Sano K, Teasdale G, et al: Report of World Federation of Neurological Surgeons Committee on a Universal Subarachnoid Hemorrhage Grading Scale. J Neurosurg 68:985-986, 1988

9. Dumont AS, Dumont RJ, Chow MM, Lin CL, Calisaneller $\mathrm{T}$, Ley KF, et al: Cerebral vasospasm after subarachnoid hemorrhage: putative role of inflammation. Neurosurgery 53:123-135, 2003

10. El-Karef A, Yoshida T, Gabazza EC, Nishioka T, Inada H, Sakakura T, et al: Deficiency of tenascin-C attenuates liver fibrosis in immune-mediated chronic hepatitis in mice. J Pathol 211:86-94, 2007

11. Fisher CM, Kistler JP, Davis JM: Relation of cerebral vasospasm to subarachnoid hemorrhage visualized by computerized tomographic scanning. Neurosurgery 6:1-9, 1980

12. Fujimoto M, Shiba M, Kawakita F, Liu L, Shimoji N, Imanaka-Yoshida K, et al: Deficiency of tenascin-C and attenuation of blood-brain barrier disruption following experimental subarachnoid hemorrhage in mice. J Neurosurg 124:1693-1702, 2016

13. Fujinaga K, Onoda K, Yamamoto K, Imanaka-Yoshida K, Takao M, Shimono T, et al: Locally applied cilostazol suppresses neointimal hyperplasia by inhibiting tenascin-C synthesis and smooth muscle cell proliferation in free artery grafts. J Thorac Cardiovasc Surg 128:357-363, 2004

14. Grieco D, Porcellini A, Avvedimento EV, Gottesman ME: Requirement for cAMP-PKA pathway activation by M phasepromoting factor in the transition from mitosis to interphase. Science 271:1718-1723, 1996

15. Hamada K, Miura Y, Toma N, Miyamoto K, Imanaka-Yoshida K, Matsushima S, et al: Gellan sulfate core platinum coil with tenascin-C promotes intra-aneurysmal organization in rats. Transl Stroke Res 5:595-603, 2014

16. Kalembeyi I, Inada H, Nishiura R, Imanaka-Yoshida K, Sakakura T, Yoshida T: Tenascin-C upregulates matrix metalloproteinase-9 in breast cancer cells: direct and synergistic effects with transforming growth factor beta1. Int J Cancer 105:53-60, 2003

17. Kasuya H, Weir B, Shen Y, Hariton G, Vollrath B, Ghahary A: Procollagen types I and III and transforming growth factor-beta gene expression in the arterial wall after exposure to periarterial blood. Neurosurgery 33:716-722, 1993

18. Khalil N, Bereznay O, Sporn M, Greenberg AH: Macrophage production of transforming growth factor beta and fibroblast collagen synthesis in chronic pulmonary inflammation. J Exp Med 170:727-737, 1989 
19. Kitazawa K, Tada T: Elevation of transforming growth factor-beta 1 level in cerebrospinal fluid of patients with communicating hydrocephalus after subarachnoid hemorrhage. Stroke 25:1400-1404, 1994

20. Massicotte EM, Del Bigio MR: Human arachnoid villi response to subarachnoid hemorrhage: possible relationship to chronic hydrocephalus. J Neurosurg 91:80-84, 1999

21. Mathiesen T, Andersson B, Loftenius A, von Holst H: Increased interleukin-6 levels in cerebrospinal fluid following subarachnoid hemorrhage. J Neurosurg 78:562-567, 1993

22. Motohashi O, Suzuki M, Shida N, Umezawa K, Ohtoh T, Sakurai Y, et al: Subarachnoid haemorrhage induced proliferation of leptomeningeal cells and deposition of extracellular matrices in the arachnoid granulations and subarachnoid space. Immunhistochemical study. Acta Neurochir (Wien) 136:88-91, 1995

23. Motohashi O, Suzuki M, Yanai N, Umezawa K, Shida N, Yoshimoto T: Thrombin and TGF-beta promote human leptomeningeal cell proliferation in vitro. Neurosci Lett 190:105108,1995

24. Niki T, Mori H: Phase I study of cilostazol. Safety evaluation at increasing single doses in healthy volunteers. Arzneimittelforschung 35 (7A): 1173-1185, 1985

25. Nishino A, Umegaki M, Fujinaka T, Yoshimine T: Cilostazol attenuates cerebral vasospasm after experimental subarachnoid hemorrhage. Neurol Res 32:873-878, 2010

26. Persky B, Low FN: Scanning electron microscopy of the subarachnoid space in the dog: inflammatory response after injection of defibrinated chicken erythrocytes. Anat Rec 212:307-318, 1985

27. Sajanti J, Heikkinen E, Majamaa K: Rapid induction of meningeal collagen synthesis in the cerebral cisternal and ventricular compartments after subarachnoid hemorrhage. Acta Neurochir Suppl 104:179-182, 2008

28. Senbokuya N, Kinouchi H, Kanemaru K, Ohashi Y, Fukamachi A, Yagi S, et al: Effects of cilostazol on cerebral vasospasm after aneurysmal subarachnoid hemorrhage: a multicenter prospective, randomized, open-label blinded end point trial. J Neurosurg 118:121-130, 2013

29. Shiba M, Fujimoto M, Imanaka-Yoshida K, Yoshida T, Taki W, Suzuki H: Tenascin-C causes neuronal apoptosis after subarachnoid hemorrhage in rats. Transl Stroke Res 5:238247, 2014

30. Suzuki H, Kanamaru K, Shiba M, Fujimoto M, ImanakaYoshida K, Yoshida T, et al: Cerebrospinal fluid tenascin-C in cerebral vasospasm after aneurysmal subarachnoid hemorrhage. J Neurosurg Anesthesiol 23:310-317, 2011

31. Suzuki H, Kanamaru K, Suzuki Y, Aimi Y, Matsubara N, Araki T, et al: Tenascin-C is induced in cerebral vasospasm after subarachnoid hemorrhage in rats and humans: a pilot study. Neurol Res 32:179-184, 2010

32. Suzuki H, Kinoshita N, Imanaka-Yoshida K, Yoshida T, Taki $\mathrm{W}$ : Cerebrospinal fluid tenascin- $\mathrm{C}$ increases preceding the development of chronic shunt-dependent hydrocephalus after subarachnoid hemorrhage. Stroke 39:1610-1612, 2008

33. Suzuki H, Muramatsu M, Tanaka K, Fujiwara H, Kojima T, Taki W: Cerebrospinal fluid ferritin in chronic hydrocepha- lus after aneurysmal subarachnoid hemorrhage. J Neurol 253:1170-1176, 2006

34. Suzuki M, Ogawa A, Sakurai Y, Nishino A, Venohara K, Mizoi K, et al: Thrombin activity in cerebrospinal fluid after subarachnoid hemorrhage. Stroke 23:1181-1182, 1992

35. Synek V, Reuben JR, Du Boulay GH: Comparing Evans' index and computerized axial tomography in assessing relationship of ventricular size to brain size. Neurology 26:231233, 1976

36. Tada T, Kanaji M, Kobayashi S: Induction of communicating hydrocephalus in mice by intrathecal injection of human recombinant transforming growth factor-beta 1 . J Neuroimmunol 50:153-158, 1994

37. Toma N, Imanaka-Yoshida K, Takeuchi T, Matsushima S, Iwata $\mathrm{H}$, Yoshida $\mathrm{T}$, et al: Tenascin-C-coated platinum coils for acceleration of organization of cavities and reduction of lumen size in a rat aneurysm model. J Neurosurg 103:681686,2005

38. Vale FL, Bradley EL, Fisher WS III: The relationship of subarachnoid hemorrhage and the need for postoperative shunting. J Neurosurg 86:462-466, 1997

39. Yoo AR, Koh SH, Cho GW, Kim SH: Inhibitory effects of cilostazol on proliferation of vascular smooth muscle cells (VSMCs) through suppression of the ERK1/2 pathway. $\mathbf{J}$ Atheroscler Thromb 17:1009-1018, 2010

40. Zhang Z, Nagata I, Kikuchi H, Xue JH, Sakai N, Sakai H, et al: Broad-spectrum and selective serine protease inhibitors prevent expression of platelet-derived growth factor-BB and cerebral vasospasm after subarachnoid hemorrhage: vasospasm caused by cisternal injection of recombinant plateletderived growth factor-BB. Stroke 32:1665-1672, 2001

\section{Disclosures}

The authors report no conflict of interest concerning the materials or methods used in this study or the findings specified in this paper.

\section{Author Contributions}

Conception and design: Suzuki. Acquisition of data: all authors. Analysis and interpretation of data: Suzuki, Nakatsuka, Kawakita, Toma, Sakaida. Drafting the article: Suzuki, Nakatsuka. Critically revising the article: Suzuki, Nakatsuka, Toma, Sakaida. Reviewed submitted version of manuscript: Suzuki, Nakatsuka, Yasuda, Umeda, Toma, Sakaida. Approved the final version of the manuscript on behalf of all authors: Suzuki. Statistical analysis: Suzuki, Nakatsuka. Administrative/technical/material support: Suzuki, Sakaida. Study supervision: Suzuki, Kawakita, Yasuda, Umeda, Sakaida.

\section{Correspondence}

Hidenori Suzuki, Department of Neurosurgery, Mie University Graduate School of Medicine, 2-174 Edobashi, Tsu, Mie 5148507, Japan.email: suzuki02@clin.medic.mie-u.ac.jp. 